\title{
The effect of fiber-electrode distance on single fiber action potentials
}

\author{
Benno K. van Veen, Willemien Wallinga, Liza Mast, \\ Ronald Busschers, Wim L.C. Rutten and Herman B.K. Boom \\ University of Twente \\ P.O. Box 217
}

7500 AE ENSCHEDE, The Netherlands

\begin{abstract}
The distance between active fibers and measuring electrodes as occuring during single fiber electronyomyography was assessed. SFAP amplitudes were calculated with a volume conduction model of skeletal muscle. Calculated SFAP's had smaller peak to peak voltages than the measured ones, the latter showed large spread. There is thus a discordance between experinent and theory in single fiber electro. myography.
\end{abstract}

\section{INTRODUCTION}

The ElectroMyoGram is an electrical manifestation of active muscle fiber membranes. At present, the Single Fiber Action Potential is considered as the fundamental building stone of the EMG signal. Interpretation of the shape of the SFAP has been hampered by lack of knowledge of the relation between this shape and the distance between the active fiber and the measuring electrode. The effect of recording distances has been estimated [1] by comparing different SFAPs measured by multielectrodes with known, fixed distances between the measuring sites. Absolute distances to the active fiber, however, remained unknown.

A technique to locate both the active fiber and the recording electrode site has been described [2]. It was applied to a limited set of SFAPs and yielded results that were hard to interpret.

SFAP distance relations can be analysed by the application of models of volume conduction in skeletal muscle. One such a model takes into account the fiber-like structure of the muscle, together with the influence of structural inhomogeneities [3]. The present paper reports on measurements of SFAP parametcrs from aclive fibers of rat EDL at known distances. The values were compared with predictions of the model described in [3]. It turned out that model predicted SFAP amplitudes were much lower than measured.

\section{METHODS}

Fiber-electrode distances were determined by marking both the active fiber and the electrode positions directly following the measurement of a SFAP. Single fibers of rat EDL (Pentobarbital anesthesia) were activated by the breaking of hyperpolarizing current pulses (duration $40 \mathrm{~ms}$ ) intracellularly delivered by way of a micropipette.

0-7803-0785-2/92\$03.00 @IEEE
This electrode also marked the fiber by iontophoresis into the fiber of the dye Lucifer Yellow CH [4]. The SFAPs were measured by a set of up to 14 wire electrodes, with electrode diameters of $25 \mu \mathrm{m}$ [4]. Electrophoretic deposition of silver from the tips of those electrodes in the set which had recorded acceptable SFAP afterwards marked their positions. After freezing of the muscle and sectioning into slices of $10 \mu \mathrm{m}$, Lucifer Yellow showed up by autofluorescence (wavelength $540 \mathrm{~nm}$ ). Bright field reflection microscopy revealed the silver depositions.
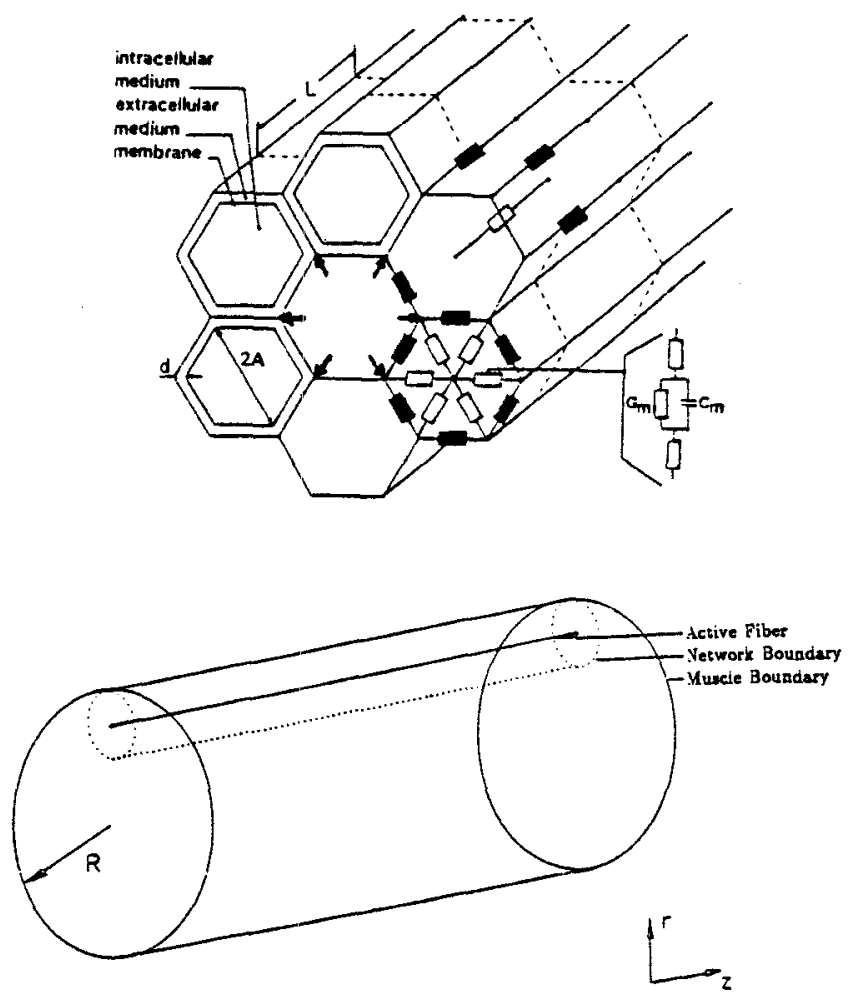

Fig. 1. Skeletal muscle model used for calculating were SFAPs. Upper panel: fiber model close to active fiber. Lower panel: surrounding continuous model

SFAPs were computed with a model of volume conduction in skeletal muscle tissue [3]. In the direct vicinity of the active fiber muscle fibers were represented by hexagonal elongated "prisms". At greater distances a homogeneous medium was assumed (Fig.1). Suitable boundary conditions ensured a correct transition from the fibrous to the homogeneous part of the model (Fig. 1). A source for the SFAP 
was described as a current-time function according to [5]. The correctness of the model was checked by comparison with an analytical, homogeneous model, applied to simple cases. Parameters in the model were taken as averages from literature. They included intracellular and extracellu lar conductivities, fiber membrane conductance and capacitance, as well as geometrical parameters.

\section{RESULTS}

Lucifer Yellow was detected in the labeled fibers over lengths from $100 \mu \mathrm{m}$ up to $3 \mathrm{~mm}$. Storage of the fiber at low temparature did not deteriorate the confinement of the dye in the fiber. Silver depositions from the measuring electrodes varied between $50 \mu \mathrm{m}^{2}$ and 200 $\mu \mathrm{m}^{2}$ and could be detected in one or, sometimes, two adjacent slices. In 25 recordings, out of 16 experiments the fiber-electrode distance could be assessed. Fig.2 shows a comparison of experimental results with the model computations. It demonstrates two striking findings. First, experimental points are widely scattered and in some cases even represent a dependance in a direction contrary to qualitative expectation. In some, at greater distances, SFAP amplitudes are greater instead of smaller. These results indicate that SFAP amplitudes are only weakly related to fiber-electrode distance. The second finding is that model calculated SFAP amplitudes are systematically much too low. This error is up to a factor 20, a value that cannot be compensated for by a simple adaptation of model parameter values.

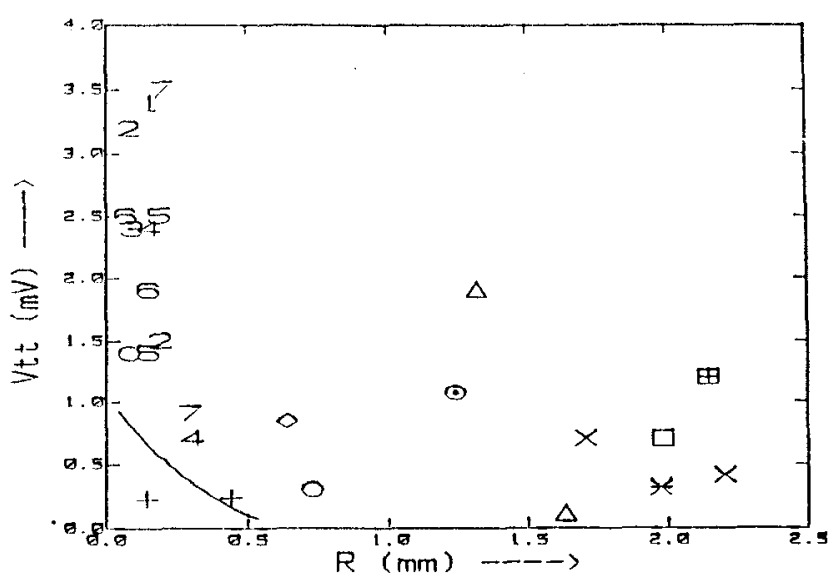

Fig. 2. Peak to peak voltages of SEAPs. Equal symbols refer to one experiment. Numbers 1-7: earlier results from [4]. The solid line is a model result.

\section{DISCUSSION}

The high scattering of SFAP amplitudes at comparable recording distances was not expected. Ekstedt [1] reported smoothly declining SFAP amplitudes on his multielectrodes. Only [6] showed, beside high, comparable values, occasional multiple maxima, on different electrodes in his needle array, implying scattered SFAP amplitudes.

The present results leave us with a dilemma: either the model or the data are in appreciable error (or both). The numerical correctness of the model was checked extensively and may be considered ascertained. The model, furthermore, assumes a more regular structure than real muscle shows. It is, however, difficult to understand how such a regularity could cause deviations of the magnitude found experimentally, especially at greater fiber-electrode distances. Inhomogeneities, especially very near to the fiber, or near to the electrode could give rise to large deviations, which, however, would be expected to be randomly positive and negative, contrary to our findings.

Noise could give rise to systematically too high amplitudes but can be excluded since all SFAP's presented in fig. 1 were inspected and found to be sufficiently noise free.

Incorrect fiber or electrode localizations could give rise to errors of the magnitude found. Identifying active fiber locations from slice to slice is a tedious task. Fibers have to be followed all the way from the stimulation electrode over thousands of muscle slices. When this procedure was carried out by at least two persons independently, the results were identical. Moreover, it is improbable that errors would occur so generally.

The processing of the muscle could have caused the silver depositions to have shifted, thus causing distance determinations. Therefore in some experiments muscles were sliced with the electrodes still in place within the muscle: the silver was seen to be deposited in the muscle, very near to the tip of the electrode in 8 of 9 tests, here no shifting of the deposition being visible.

The current results suggest, that, between active muscle fibers and (small) measuring electrodes, conducting pathways exist that were not accounted for in existing volume conduction models, including ours.

\section{REFERENCES}

[1] J. Ekstedt. "Human single muscle fiber action potentials," Acta Physiol. Scand., 61 (Suppl.226). 1964.

[2] W. Wallinga, B.A. Albers, J.H.M. Put, W.L.C. Rutten, P. Wirtz, "Acivity of single muscle fibers at known distances". In: Electrophysiological kinesiology; editors W. Wallinga, H.B.K. Boom, J.de Vries, 1988, pp.221-224, Elseviers Science Publishers B.V., Amsterdam.

[3] B.K. van Veen. "Single fiber action potentials in inhomogeneously conducting sketetal muscle". Ph.D. thesis. 1992, University of Twente, The Netherlands.

[4] B.A. Albers, J.H.M. Put, W. Wallinga, P. Wirtz, "Quantitative analysis of single muscle fibre action porentials recorded at known distances", Electroenceph. and clin. Neurophysiol. 73, 245-253, 1989.

[5] P. Rosenfalck. "Intra- and extracellular potential fields of active nerve and muscle fibers. A physicomathematical analysis of different models", Akademisk Forlag. Copenhagen, 1969.

[6] T.H.J.M. Gootzen. "Muscle fiber and motor unit action potentials," Ph.D. thesis. 1990. University of Nijmegen, The Netherlands. 